\title{
Fully Biobased Highly Transparent Nanopaper with UV-Blocking Functionality
}

\author{
Douglas R. Hayden, ${ }^{*},, \| \odot$ Srivatssan Mohan, ${ }^{\dagger, \|}$ Arnout Imhof, ${ }^{*} \dagger \odot$ and Krassimir P. Velikov ${ }^{\dagger, \ddagger, \S}$ \\ ${ }^{\dagger}$ Soft Condensed Matter, Debye Institute for Nanomaterials Science, Utrecht University, Princetonplein 1, 3584 CC Utrecht, The \\ Netherlands \\ ${ }^{\ddagger}$ Unilever R\&D Vlaardingen, Olivier van Noortlaan 120, 3133 AT Vlaardingen, The Netherlands \\ ${ }^{\S}$ Institute of Physics, University of Amsterdam, Science Park 904, 1098 XH Amsterdam, The Netherlands
}

Supporting Information

ABSTRACT: The development of green alternatives for petroleumbased plastics is essential for the protection of our environment and its ecosystems. Here, we demonstrate broadband UV-blocking, highly transparent composite nanopaper films from a waste source of cellulose nanofibrils with embedded tunable UV-absorbing nanoparticles (NPs) from ethyl cellulose. These functional nanopaper films are highly transparent, selectively block UV light, and show excellent photostability, therefore with great potential as highperformance, renewable, sustainable, and biodegradable materials for photoprotection applications. Moreover, the integration of

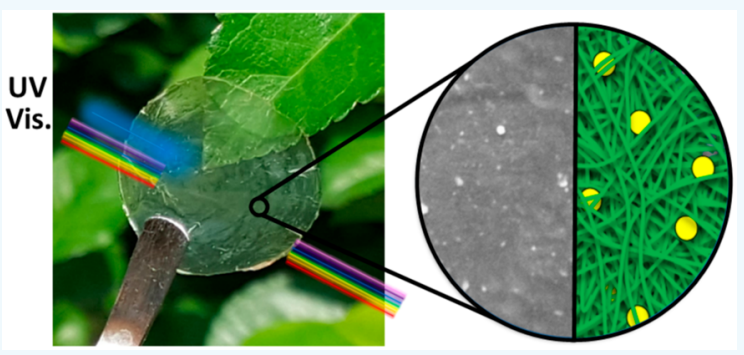
functionalized NPs from ethyl cellulose into nanopaper is a platform for novel advanced sustainable materials with a myriad of functionality.

KEYWORDS: biobased, photoprotection, nanopaper, ethyl cellulose nanoparticles, nanocellulose

$\mathrm{T}$ he problem of plastic waste is one of the greatest challenges faced by the current generation. Every year, millions of tons of plastic pollutes our oceans resulting in potentially major damage to marine life, biodiversity, food security, and human health. The development of renewable, sustainable, and biodegradable alternatives for plastic materials is therefore essential.

Nanopaper has attracted attention as a low-cost, environmentally friendly, high-performance material with strong potential to replace plastic substrates in many electronic and material applications. ${ }^{1-4}$ Nanopaper is prepared from the same chemical constituents as regular paper but uses very thin cellulose nanofibrils $(<20 \mathrm{~nm})$ instead of thicker fibers. These very thin cellulose nanofibrils can advantageously be obtained from diverse sources (multiple plants or bacteria) including waste sources, instead of thicker fibers that are typically obtained from wood pulp. Preparing a material from these thinner nanofibrils results in a material that exhibits superior mechanical and barrier properties to regular paper, and significantly, is also optically transparent. ${ }^{1,4,3}$ The transparent property arises because nanofibrils are much less effective in scattering visible light than larger cellulose fibrils, and can also pack together more efficiently. ${ }^{1,3}$ The transparent nature of nanopaper has opened up the potential for optically functionalized paper-based materials.

Paper-based materials like nanopaper are excellent substrates for functionalization by nanoparticles (NPs) because the porous structure allows for high NP loadings. ${ }^{6}$ Additionally, paper-based materials can be effectively functionalized by a wide variety of NPs, resulting in materials suitable for a wide range of applications. For example, the use of inorganic NPs (e.g., $\mathrm{TiO}_{2}, \mathrm{Au}, \mathrm{Ag}$ ) can produce paper-based materials with excellent catalytic, ${ }^{7}$ antibacterial, ${ }^{8}$ sensing, ${ }^{9}$ and anticounterfeit ${ }^{10}$ properties. Despite the easy functionalization of regular paper-based materials with inorganic NPs, the opaque nature of regular paper limits the potential for optical functionalization. Moreover, inorganic NPs are (i) non-biobased which limits the renewable and sustainable nature of the resultant material, and (ii) unable to easily utilize functional organic molecules. Therefore, there is a need to develop materials from transparent nanopaper which can be functionalized with biobased organic NPs containing functional organic molecules as encapsulants-which offer a great range of functionality.

Here, we develop transparent UV-blocking nanopaper by embedding tunable UV-absorbing NPs from ethyl cellulose ${ }^{11}$ into nanopaper. The embedded NPs are used as carriers for two types of UV-absorbing species: (i) organic UV filters commonly used in sunscreens (oxybenzone, octinoxate, and avobenzone), and (ii) biobased UV-absorbing molecules from plants (quercetin, retinol, and p-coumaric acid). Significantly, the nanopaper prepared with the latter set of embedded NPs is entirely biobased. Both the NPs and nanopaper preparation processes are upscalable, and moreover, we adopt a more

Received: February 28, 2019

Accepted: March 7, 2019

Published: March 7, 2019 


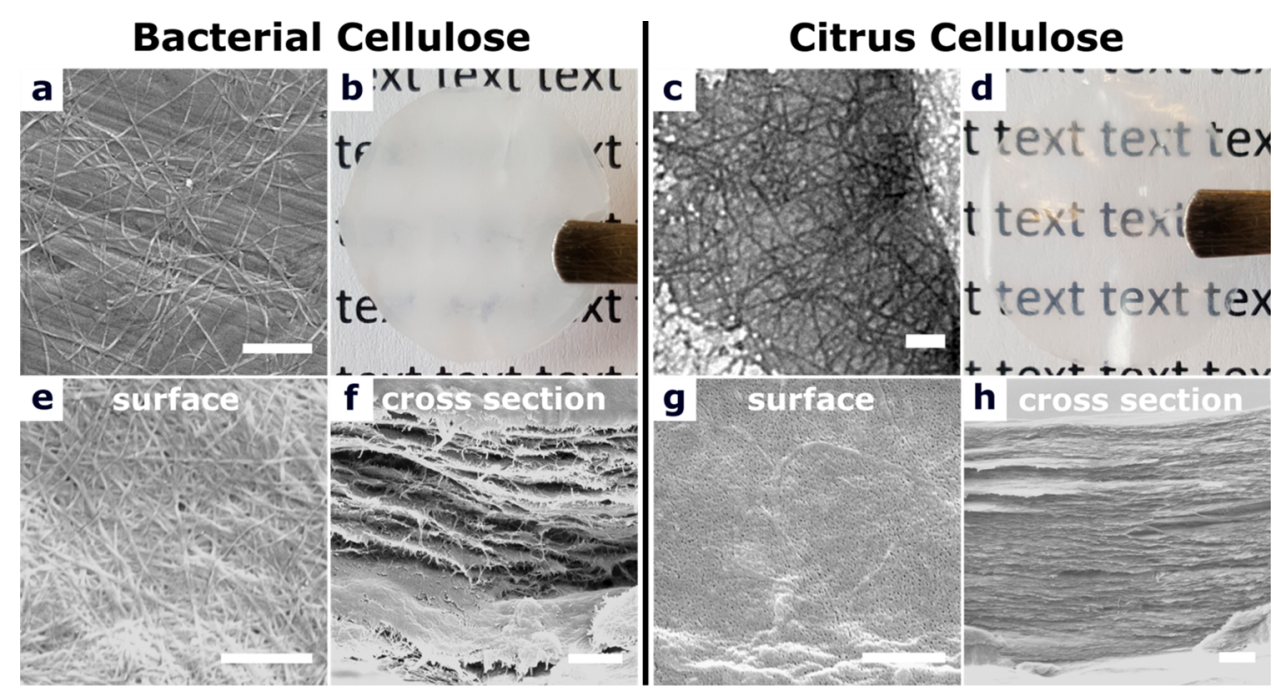

Figure 1. (a, c) SEM and TEM images, respectively, of the oxidized bacterial and (negatively stained) citrus cellulose nanofibrils. (b, d) Photos of the nanopaper films prepared from bacterial $(\mathrm{b})$ and citrus $(\mathrm{d})$ cellulose sources, $1 \mathrm{~cm}$ away from the text. (e-h) SEM images of the surface and cross section of the nanopaper film from bacterial (e, f) and citrus (g, h) cellulose sources. Scale bars: (a, e, g) $1 \mu \mathrm{m},(\mathrm{c}) 100 \mathrm{~nm}$, and (f, h) $2 \mu \mathrm{m}$.

environmentally friendly route to prepare our nanopaper by retaining the fibrillar structure of cellulose instead of dissolving it using toxic solvents (dissolution is often performed to prepare highly transparent cellulose-based materials, i.e., cellophane and nanopaper containing cellulose nanocrystals (CNCs)). ${ }^{5,12,13}$ Effective UV blocking cellulose films can also be prepared by incorporating inorganic $\mathrm{ZnO}^{14,15}$ or synthetic fibers ${ }^{16}$ into nanopaper, or even using chemically modified UV-absorbing CNCs as fillers in a polymeric matrix, ${ }^{17}$ but the resulting materials are all nonbiobased and the latter two examples involve toxic solvents. Fully biobased UV-blocking cellulose films can be prepared by incorporating lignincontaining cellulose nanofibrils into nanopaper, ${ }^{12,18}$ but this technique significantly compromises film appearance and transparency. In our method, the incorporation of biobased UV-absorbing NPs advantageously allows us to more selectively block UV light while maintaining excellent transparency and appearance of the films. We envisage that our transparent UV-blocking nanopaper has great potential for low-cost, high-performance, sustainable materials in a wide variety of photoprotection applications including food and beverage packaging, eye-protection, consumer goods packaging, photoresist templates, UV protective materials for solar panels, and UV filter window shades. Even though we focus here on the potential of transparent UV protective materials, the NPs we use are potential vehicles for a vast range of organic molecules with a variety of properties, including: dyes, ${ }^{19}$ fragrances, ${ }^{20}$ antimicrobial, ${ }^{21}$ photochromic molecules, $^{22}$ and potentially many more. Therefore, the novel principle of functionalizing nanopaper with size and compositionally tunable ethyl cellulose $\mathrm{NPs}^{23}$ allows for the facile fabrication of environmentally friendly materials with a potentially almost unlimited functionality, as an alternative to plastics in a tremendous range of applications.

We first prepared simple nonfunctionalized nanopaper using cellulose nanofibrils from two sources: (i) bacteria and (ii) primary cell walls in citrus peels waste after production of pectin. The fibrils from both sources are a few tens of nanometers in width in their native state, ${ }^{24}$ unlike in the case of wood pulp where extensive mechanical/mechanochemical treatment needs to be applied to obtain fibrils in the nanometer range. However, in a dispersed state, these fibrils have a tendency to aggregate due to their attractive van der Waals and hydrogen-bonding interactions. ${ }^{25}$ Since the crosssectional dimension of the fibrils plays a crucial role in fabricating nanopaper, it is important to individualize the nanofibrils in the dispersed state. This was done by a combination of a TEMPO-mediated surface oxidation reaction $^{26}$ and a high-energy mechanical deagglomeration process. $^{25}$ The oxidation reaction results in the conversion of some hydroxyl groups to charged carboxyl groups at the fibril surface leading to electrostatic stabilization of the nanofibrils against agglomeration. Both cellulose sources underwent the same individualization process, which disintegrates the fibrils into much thinner elementary fibril components (nanofibrils are composed of bundles of elementary fibrils with a width of $\left.\sim 4 \mathrm{~nm}^{1,25}\right)$. The structure of fibrils obtained after the oxidation reaction is known to be strongly dependent on the starting material, ${ }^{27}$ and indeed we found that the width of cellulose nanofibrils from bacteria remained similar after the individualization process (mean width $43 \mathrm{~nm}$, Figure 1a and discrete size distributions shown in Figure S5), which has been reported previously, ${ }^{28}$ while the individualization process on citrus cellulose yielded much thinner nanofibrils with a mean width of $7 \mathrm{~nm}$ (Figure 1c and discrete size distributions shown in Figure S5), close to that of elementary fibrils.

We prepared nanopaper from both cellulose sources via a simple vacuum filtration followed by gentle heating and pressing. Remarkably, the nanopaper prepared using cellulose nanofibrils from the citrus source was significantly more transparent and less hazy than the nanopaper prepared using the nanofibrils from bacterial cellulose (Figure $1 \mathrm{~b}$ vs $d$ ), at a similar thickness $(8-12 \mu \mathrm{m}$ as measured by SEM imaging of cross sections in Figure 1). We hypothesize that the higher transparency of the nanopaper prepared from citrus cellulose nanofibrils arises because these nanofibrils are much thinner than the cellulose nanofibrils from the bacterial source, despite both cellulose sources undergoing the same individualization process. These thinner nanofibrils result in more transparent nanopaper films because they scatter visible light less effectively than the thicker nanofibrils (Rayleigh's scattering theory predicts $\sigma_{\text {set }} \propto D^{4}$ for long thin fibers, where $\sigma_{\text {set }}$ is the 

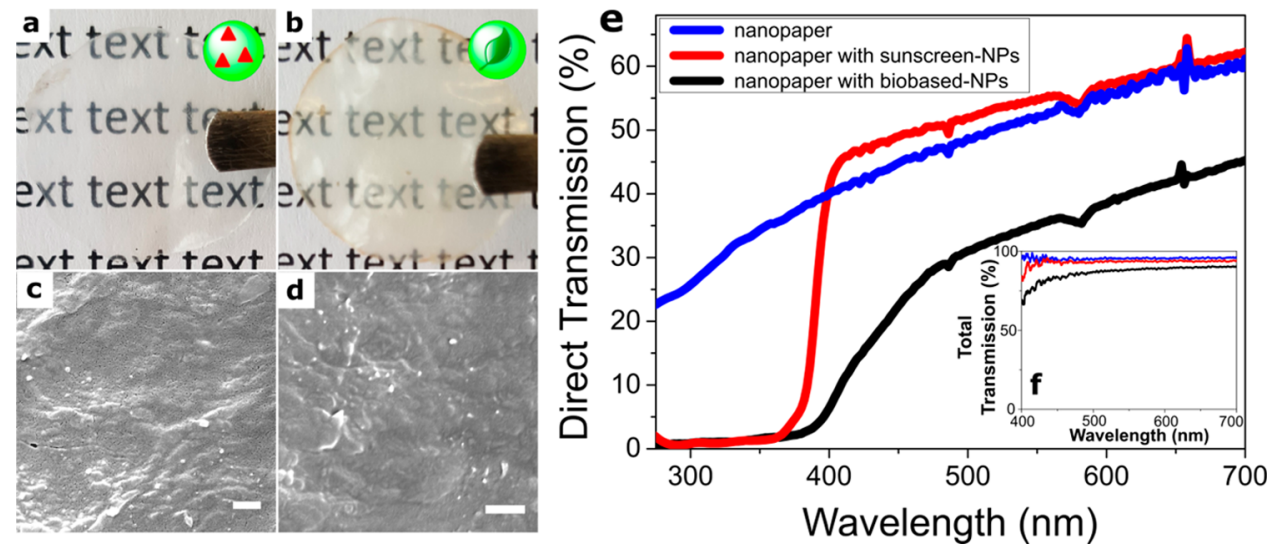

Figure 2. (a, b) Photos of nanopaper films functionalized with (a) sunscreen-NPs and (b) biobased-NPs, $1 \mathrm{~cm}$ away from underlying text. (c, d) SEM images of the surface of the nanopaper films (a) and (b), respectively, in which sunscreen-NPs and biobased-NPs are visible on the respective surfaces. Both scale bars are $500 \mathrm{~nm}$. (e) Spectral transmission measurements for the three films. (f) Total transmission measurements for the three films.

scattering cross section and $D$ is the fiber diameter ${ }^{3}$ ) and can also pack together more efficiently. The denser packing of nanofibrils results in fewer air gaps within the cellulose film and therefore less haze, ${ }^{4}$ which arises as a result of the refractive index mismatch between the cellulose and structural air gaps causing light scattering. Moreover, nanopaper prepared with thinner fibrils is reported to have better mechanical properties than nanopaper prepared with thicker fibrils, by an anomalous scaling law. ${ }^{29}$ The difference in nanofibrils size between the bacterial and citrus cellulose sources is also apparent when observing the nanopaper film surface in Figure 1, where the individual nanofibrils are distinguishable on the surface of the bacterial cellulose nanopaper (Figure 1e), but indistinguishable on the surface of the citrus cellulose nanopaper (Figure 1g). We therefore found that this citrus waste cellulose source is very beneficial for preparing highly transparent nanopaper.

Consequently, we chose to use cellulose nanofibrils from the citrus source, instead of bacterial, to prepare transparent UVblocking nanopaper. We prepared these films by premixing the cellulose nanofibrils with UV-absorbing ethyl cellulose NPs before proceeding with the same filtration, heating, and pressing technique used for the preparation of the pure nanopaper. We used two types of UV-absorbing ethyl cellulose NPs: (i) "sunscreen-NPs" containing encapsulated commonplace organic UV filters from cosmetic sunscreens (oxybenzone, avobenzone, and octinoxate), ${ }^{11,23}$ and (ii) "biobasedNPs" containing encapsulated biobased UV-absorbing molecules obtainable from plants (quercetin, retinol, and pcoumaric acid). ${ }^{19}$ Both the sunscreen-NPs and biobased-NPs were prepared by an upscalable "antisolvent precipitation" technique, and were prepared using 10 and 34 wt \% UVabsorbing material respectively in the antisolvent precipitation because these recipes are known to give stable aqueous NP dispersions and provide effective uniform absorbance across the entire UV spectrum. ${ }^{11,19,23}$ The resultant prepared sunscreen-NPs and biobased-NPs show average particle diameters of 70 and $90 \mathrm{~nm}$, respectively (DLS measurements in Figure S1 and SEM images in Figure S2), and demonstrate effective uniform absorbance across the entire UV spectrum $\lambda$ $=290-400 \mathrm{~nm}$ (Figure S3). ${ }^{11,23}$ The biobased-NPs also absorb up to $\lambda=450 \mathrm{~nm}$ due to the broad absorbance profile of quercetin, resulting in a yellow appearance (Figure S4).
We found that the nanopaper containing the sunscreen-NPs is almost identical in appearance to the nonfunctionalized nanopaper (Figure 1d vs Figure 2a), demonstrates similar direct and total transmission values in the visible spectrum (Figure 2e,f), but completely blocks out UV light $(<1 \%$ average between $\lambda=290-380 \mathrm{~nm}$, Figure 2e). The nanopaper containing the biobased-NPs is also similar in appearance to the other two films, completely blocks out UV light (Figure $2 \mathrm{e})$, but has a slight yellow tinge (Figure $2 \mathrm{~b}$ ) due to the presence of quercetin in the biobased-NPs. This fully biobased nanopaper also appears slightly hazier than the other films, which is confirmed by slightly lower direct and diffuse transmission values (Figure 2e,f). This is likely due to the increased scattering caused by the slightly larger biobased-NPs compared to the sunscreen-NPs, especially considering that the thickness and root-mean-square (rms) surface roughness for both films are very similar to each other, where the thickness is $9.5 \pm 0.4$ and $9.6 \pm 0.3 \mu \mathrm{m}$ for the films functionalized with sunscreen-NPs and biobased-NPs, respectively (Figure S7a,b), and the rms surface roughness is 67 and $68 \mathrm{~nm}$, respectively (Figure S8). Improving the transparency of nanopaper can be achieved with more advanced nanopaper preparation methods and techniques. For example, postprocessing techniques such as polishing, ${ }^{1}$ heat pressing, ${ }^{3}$ and irreversible collapsing by capillary action by the evaporation of water during pressing are all known to result in far greater transparency. We therefore believe that the transparency/haziness of these films could be improved further by such postprocessing techniques. Despite this, we find that our films are highly transparent (direct transmission $51 \%, 57 \%$, and $39 \%$ and total transmission $95 \%$, $94 \%$, and $89 \%$ at $\lambda=600 \mathrm{~nm}$ for nanopaper, nanopaper with sunscreen-NPs, and nanopaper with biobased-NPs respectively), with far greater transparency values than UV blocking films containing lignin. ${ }^{12,18}$ Moreover, the haze $\left(H_{\mathrm{T}}\right)$ values of these films $(47 \%, 39 \%$, and $56 \%$ respectively at $\lambda=600 \mathrm{~nm})$ are lower than haze values for typical (not postprocessed) nanopaper $(>60 \%$ at $\lambda=600 \mathrm{~nm}), 5,30$ as determined by ${ }^{5}$

$$
H_{\mathrm{T}}=100 \times \frac{(\text { total transmission }- \text { direct transmission })}{\text { total transmission }}
$$

This is remarkable considering we use such a simple technique of nanopaper production. 


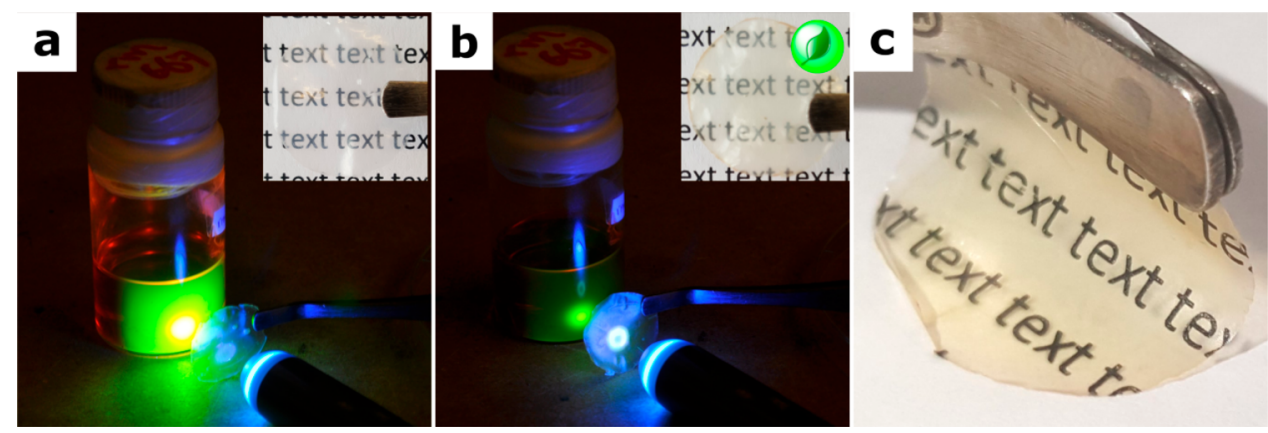

Figure 3. ( $\mathrm{a}, \mathrm{b}$ ) Photos of a glass vial containing $2 \mathrm{~nm}$ CdSe quantum dots which fluoresce as a result of stimulation by a $\lambda=375 \mathrm{~nm}$ laser light. In (a), the UV laser light is shined through the nonfunctionalized nanopaper (from Figure 1d, shown in the inset) and the quantum dots are strongly fluorescing, whereas in (b) the UV laser is shined through the nanopaper functionalized with biobased-NPs (from Figure 2b, shown in the inset) and the fluorescence is significantly suppressed. (c) Photo of nanopaper functionalized with biobased-NPs with text printed on, being bent to demonstrate flexibility.

The presence of the NPs in both the resultant UV-blocking films is supported by SEM imaging of the surface (Figure $2 \mathrm{c}$ and $\mathrm{d}$ ) and cross section (Figure S7c). In order to achieve complete UV-blockage, we used a mass ratio of 44:56 [NPs/ nanofibrils] for the preparation of the films. We did find that some particles were lost upon preparing the films via vacuum filtration, but this was only a small amount: $\sim 95 \%$ of both the added sunscreen-NPs and biobased-NPs were retained in the resultant films respectively (meaning the other 5\% passed through the filter), which we determined from the absorbance of the filtrate. Improving the transparency of the films (e.g., with postprocessing techniques) may result in more NPs required for complete UV blockage than what we use, because more transparent films will typically have greater transmission of UV light too, therefore requiring more NPs in order to fully block UV radiation. In this case, both (i) the amount of NPs embedded in the film as well as (ii) the loading of the UVabsorbing molecules into the NPs, can simply be tuned either by varying the amount of NPs used in the nanopaper preparation or by varying the amount of UV-absorbing material in the NP preparation (also known as antisolvent precipitation) process, in order to provide the desired UV protection. As a visual demonstration of the UV-blocking ability of the fully biobased UV-blocking nanopaper, we show that the fluorescence of CdSe quantum dots, which fluoresce on exposure to a $\lambda=375 \mathrm{~nm}$ UV laser, is significantly suppressed when the UV laser is shined through our UVblocking nanopaper, as compared to the nonfunctionalized nanopaper (Figure 3a,b). Moreover, we demonstrate the flexibility and ability to print onto the fully biobased UVblocking nanopaper in Figure 3c. We observed no obvious difference in the mechanical properties of the UV-blocking films compared to the nonfunctionalized film, where all films are flexible and can be repeatedly bent without damage.

The UV-blocking films demonstrate effective photostability as a function of time, maintaining identical appearance (Figure $4 \mathrm{a}-\mathrm{c}$ ) and transparency (Figure $4 \mathrm{~d}$, transmission of visible light $\lambda=400-700 \mathrm{~nm}$ is almost identical to Figure $2 \mathrm{e}$ ) when exposed to sunlight over a period of 5 months on a windowsill. This duration and intensity of sunlight exposure can be compared, for example, to many food packaging materials in supermarkets. The UV-blocking ability of both the nanopaper with sunscreen-NPs and nanopaper with biobased-NPs remained effective but did degrade slightly, in which the films show average direct transmission values of $10 \%$ and $9 \%$,

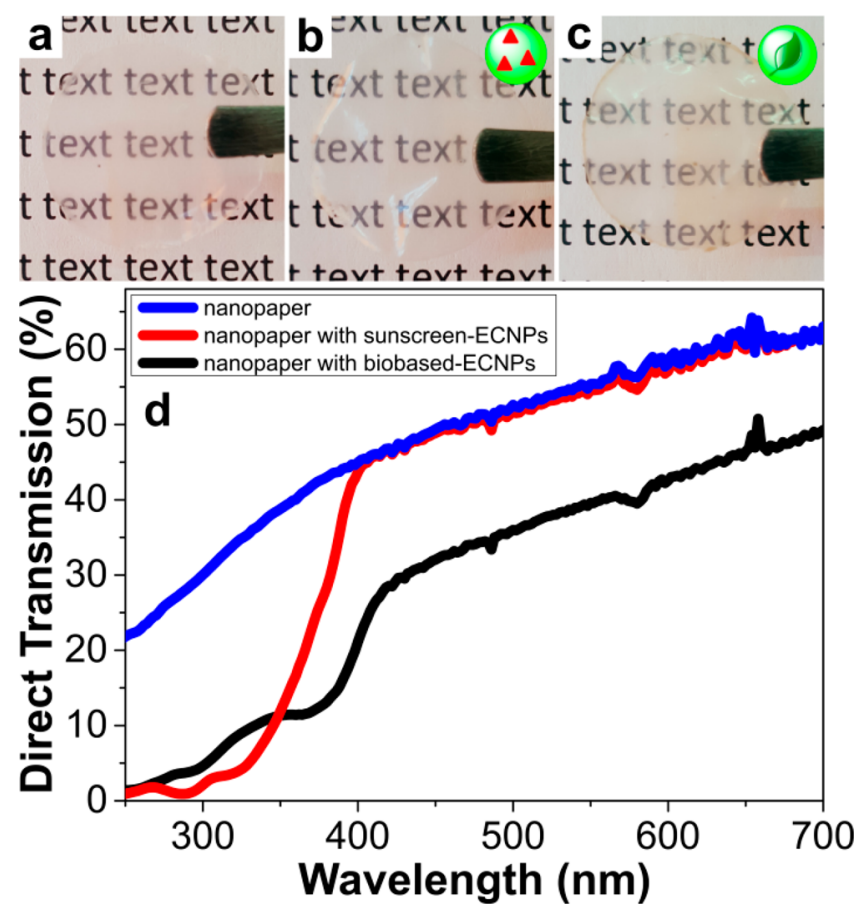

Figure 4. $(\mathrm{a}-\mathrm{c})$ Photos and $(\mathrm{d})$ transmission measurements of the three films from Figure 2 after 5 months of sunlight exposure. (a) Nanopaper, (b) nanopaper with embedded sunscreen-NPs, and (c) fully biobased nanopaper with embedded biobased-NPs.

respectively (between $\lambda=290-380 \mathrm{~nm}$ ), as opposed to $<1 \%$ before sunlight exposure. Despite this, even after the extensive sunlight exposure, these values are still much lower than that of the nonfunctionalized nanopaper which transmits 36\% (Figure $4 d)$. Since the UV absorption is defined by the degradation of the compounds encapsulated inside the NPs, ${ }^{19,23}$ the photostability of both films could be vastly improved simply by loading the films with more NPs or loading the NPs with more UV-absorbing compounds, so that the films maintain full UV blockage despite degradation of the UV-absorbing compounds. Additionally, the UV-blocking films also demonstrated thermal stability very similar to typical nanopaper (Figure S9).

In conclusion, we prepared highly transparent UV-blocking nanopaper with embedded UV-absorbing NPs from ethyl cellulose, via a completely upscalable technique. We use a waste source of cellulose nanofibrils resulting in highly transparent films. We then prepare nanopaper which selectively 
blocks UV light without compromising film quality by embedding sunscreen-NPs. Significantly, we also prepare transparent UV-blocking nanopaper with a fully biobased composition by embedding biobased-NPs with encapsulated plant-based UV-absorbing compounds. The films blocked UV light completely $(<1 \%$ direct transmission between $\lambda=290-$ $380 \mathrm{~nm}$ ) while exhibiting high transparency (57\%/94\% and $39 \% / 89 \%$ direct/total transmission at $\lambda=600)$, low haze $(39 \%$ and $56 \%$ at $\lambda=600$ ), and good thermal and photostability (still $<10 \%$ direct transmission between $\lambda=290-380 \mathrm{~nm}$ after 5 months of sunlight exposure). Crucially, these films are entirely upscalable and the embedded ethyl cellulose NPs are potential vehicles for a huge range of organic molecules, with a wide range of functionality. This system therefore is highly promising for the innovation of novel and advanced sustainable functional materials for many applications.

\section{ASSOCIATED CONTENT}

\section{S Supporting Information}

The Supporting Information is available free of charge on the ACS Publications website at DOI: 10.1021/acsapm.9b00192.

Materials and methods, further characterization of the NPs, nanofibrils, UV-blocking nanopaper from citrus cellulose, and UV-blocking nanopaper from bacterial cellulose (PDF)

\section{AUTHOR INFORMATION}

\section{Corresponding Authors}

*E-mail: d.r.hayden@uu.nl.

*E-mail: a.imhof@uu.nl.

\section{ORCID}

Douglas R. Hayden: 0000-0001-7380-6054

Arnout Imhof: 0000-0002-7445-1360

Krassimir P. Velikov: 0000-0002-8838-1201

\section{Author Contributions}

"D.R.H. and S.M. contributed equally.

Notes

The authors declare no competing financial interest.

\section{ACKNOWLEDGMENTS}

This research is supported by the Dutch Technology Foundation STW (Grant No. 13567), which is part of The Netherlands Organization for Scientific Research (NWO), and which is partly funded by the Ministry of Economic Affairs, as well as the Foundation for Fundamental Research on Matter (FOM), financially supported by a FOM program grant (Grant No. 143). We thank Chris Schneijdenberg and Dave van den Heuvel for technical assistance, Roland Gouzy for his assistance in processing the nanofibril dispersions, Relinde J. A. van Dijk-Moes for providing the quantum dots in Figure ${ }^{3,}$, Heleen Kibbelaar for providing the biobased-NPs, L. Christiaan Post for AFM measurements, J. Lennart Weber for TGA measurements, and Tonnishtha Dasgupta for critical reading of the manuscript.

\section{REFERENCES}

(1) Nogi, M.; Iwamoto, S.; Nakagaito, A. N.; Yano, H. Optically Transparent Nanofiber Paper. Adv. Mater. 2009, 21, 1595-1598.

(2) Lin, Y.; Gritsenko, D.; Liu, Q.; Lu, X.; Xu, J. Recent Advancements in Functionalized Paper-Based Electronics. ACS Appl. Mater. Interfaces 2016, 8, 20501-20515.
(3) Zhu, H.; Parvinian, S.; Preston, C.; Vaaland, O.; Ruan, Z.; Hu, L. Transparent Nanopaper with Tailored Optical Properties. Nanoscale 2013, 5, 3787-3792.

(4) Hsieh, M. C.; Koga, H.; Suganuma, K.; Nogi, M. Hazy Transparent Cellulose Nanopaper. Sci. Rep. 2017, 7, 41590.

(5) Xu, X.; Zhou, J.; Jiang, L.; Lubineau, G.; Ng, T.; Ooi, B. S.; Liao, H. Y.; Shen, C.; Chen, L.; Zhu, J. Y. Highly Transparent, Low-Haze, Hybrid Cellulose Nanopaper as Electrodes for Flexible Electronics. Nanoscale 2016, 8, 12294-12306.

(6) Ngo, Y. H.; Li, D.; Simon, G. P.; Garnier, G. Paper Surfaces Functionalized by Nanoparticles. Adv. Colloid Interface Sci. 2011, 163, 23-38.

(7) Li, S. X.; Lin, X.; Zheng, F. Y.; Liang, W.; Zhong, Y.; Cai, J. Constituting Fully Integrated Visual Analysis System for $\mathrm{Cu}(\mathrm{II})$ on TiO2/Cellulose Paper. Anal. Chem. 2014, 86, 7079-7083.

(8) Tankhiwale, R.; Bajpai, S. K. Graft Copolymerization onto Cellulose-Based Filter Paper and Its Further Development as Silver Nanoparticles Loaded Antibacterial Food-Packaging Material. Colloids Surf., B 2009, 69, 164-168.

(9) Niarchos, G.; Dubourg, G.; Afroudakis, G.; Tsouti, V.; Makarona, E.; Matović, J.; Crnojević-Bengin, V.; Tsamis, C. PaperBased Humidity Sensor Coated with $\mathrm{ZnO}$ Nanoparticles: The Influence of $\mathrm{ZnO}$. Procedia Eng. 2016, 168, 325-328.

(10) Zhang, Y.; Aslan, K.; Previte, M. J. R.; Geddes, C. D. MetalEnhanced Fluorescence from Paper Substrates: Modified Spectral Properties of Dyes for Potential High-Throughput Surface Analysis and Assays and as an Anti-Counterfeiting Technology. Dyes Pigm. 2008, 77, 545-549.

(11) Hayden, D. R.; Imhof, A.; Velikov, K. P. Biobased Nanoparticles for Broadband UV Protection with Photostabilized UV Filters. ACS Appl. Mater. Interfaces 2016, 8, 32655-32660.

(12) Sadeghifar, H.; Venditti, R.; Jur, J.; Gorga, R. E.; Pawlak, J. J. Cellulose-Lignin Biodegradable and Flexible UV Protection Film. ACS Sustainable Chem. Eng. 2017, 5, 625-631.

(13) Yang, Q.; Fukuzumi, H.; Saito, T.; Isogai, A.; Zhang, L. Transparent Cellulose Films with High Gas Barrier Properties Fabricated from Aqueous Alkali/Urea Solutions. Biomacromolecules 2011, 12, 2766-2771.

(14) Feng, X.; Zhao, Y.; Jiang, Y.; Miao, M.; Cao, S.; Fang, J. Use of Carbon Dots to Enhance UV-Blocking of Transparent Nanocellulose Films. Carbohydr. Polym. 2017, 161, 253-260.

(15) Jiang, Y.; Song, Y.; Miao, M.; Cao, S.; Feng, X.; Fang, J.; Shi, L. Transparent Nanocellulose Hybrid Films Functionalized with $\mathrm{ZnO}$ Nanostructures for UV-Blocking. J. Mater. Chem. C 2015, 3, 67176724.

(16) Luo, J.; Zhang, M.; Yang, B.; Liu, G.; Tan, J.; Nie, J.; Song, S. A Promising Transparent and UV-Shielding Composite Film Prepared by Aramid Nanofibers and Nanofibrillated Cellulose. Carbohydr. Polym. 2019, 203, 110-118.

(17) Sirviö, J. A.; Visanko, M.; Heiskanen, J. P.; Liimatainen, H. UVAbsorbing Cellulose Nanocrystals as Functional Reinforcing Fillers in Polymer Nanocomposite Films. J. Mater. Chem. A 2016, 4, 63686375.

(18) Wang, Q.; Du, H.; Zhang, F.; Zhang, Y.; Wu, M.; Yu, G.; Liu, C.; Li, B.; Peng, H. Flexible Cellulose Nanopaper with High Wet Tensile Strength, High Toughness and Tunable Ultraviolet Blocking Ability Fabricated from Tobacco Stalk: Via a Sustainable Method. J. Mater. Chem. A 2018, 6, 13021-13030.

(19) Hayden, D. R.; Kibbelaar, H. V. M.; Imhof, A.; Velikov, K. P. Fully-Biobased UV-Absorbing Nanoparticles from Ethyl Cellulose and Zein for Environmentally Friendly Photoprotection. RSC Adv. 2018, $8,25104-25111$

(20) Sansukcharearnpon, A.; Wanichwecharungruang, S.; Leepipatpaiboon, N.; Kerdcharoen, T.; Arayachukeat, S. High Loading Fragrance Encapsulation Based on a Polymer-Blend: Preparation and Release Behavior. Int. J. Pharm. 2010, 391, 267-273.

(21) Pan-In, P.; Banlunara, W.; Chaichanawongsaroj, N.; Wanichwecharungruang, S. Ethyl Cellulose Nanoparticles: Claritho- 
mycin Encapsulation and Eradication of H. Pylori. Carbohydr. Polym.

2014, 109, 22-27.

(22) Vílchez-Maldonado, S.; Calderó, G.; Esquena, J.; Molina, R. UV Protective Textiles by the Deposition of Functional Ethylcellulose Nanoparticles. Cellulose 2014, 21, 2133-2145.

(23) Hayden, D. R.; Kibbelaar, H. V. M.; Imhof, A.; Velikov, K. P. Size and Optically Tunable Ethyl Cellulose Nanoparticles as Carriers for Organic UV Filters. ChemNanoMat 2018, 4, 301-308.

(24) Mohan, S.; Jose, J.; Kuijk, A.; Veen, S. J.; van Blaaderen, A.; Velikov, K. P. Revealing and Quantifying the Three-Dimensional Nano- and Microscale Structures in Self-Assembled Cellulose Microfibrils in Dispersions. ACS Omega 2017, 2, 5019-5024.

(25) Kuijk, A.; Koppert, R.; Versluis, P.; van Dalen, G.; Remijn, C.; Hazekamp, J.; Nijsse, J.; Velikov, K. P. Dispersions of Attractive Semi Flexible Fiberlike Colloidal Particles from Bacterial Cellulose MicroFibrils. Langmuir 2013, 29, 14356-14360.

(26) Saito, T.; Hirota, M.; Tamura, N.; Kimura, S.; Fukuzumi, H.; Heux, L.; Isogai, A. Individualization of Nano-Sized Plant Cellulose Fibrils by Direct Surface Carboxylation Using TEMPO Catalyst under Neutral Conditions. Biomacromolecules 2009, 10, 1992-1996.

(27) Siró, I.; Plackett, D. Microfibrillated Cellulose and New Nanocomposite Materials: A Review. Cellulose 2010, 17, 459-494.

(28) Saito, T.; Nishiyama, Y.; Putaux, J. L.; Vignon, M.; Isogai, A. Homogeneous Suspensions of Individualized Microfibrils from TEMPO-Catalyzed Oxidation of Native Cellulose. Biomacromolecules 2006, 7, 1687-1691.

(29) Zhu, H.; Zhu, S.; Jia, Z.; Parvinian, S.; Li, Y.; Vaaland, O.; Hu, L.; Li, T. Anomalous Scaling Law of Strength and Toughness of Cellulose Nanopaper. Proc. Natl. Acad. Sci. U. S. A. 2015, 112, 89718976.

(30) Fang, Z.; Zhu, H.; Yuan, Y.; Ha, D.; Zhu, S.; Preston, C.; Chen, Q.; Li, Y.; Han, X.; Lee, S.; Chen, G.; Li, T.; Munday, J.; Huang, J.; $\mathrm{Hu}, \mathrm{L}$. Novel Nanostructured Paper with Ultrahigh Transparency and Ultrahigh Haze for Solar Cells. Nano Lett. 2014, 14, 765-773. 